LAWRENCE LIVERMORE N A T IO N A L LABORATORY
Measurement of the absolute hohlraum wall albedo under ignition foot drive conditions

O. S. Jones, S. H. Glenzer, L. J. Suter, R. E. Turner, K. M. Campbell, E. L. DeWald, B. A. Hammel, J. H. Hammer, R. L. Kauffmann, O. L. Landen, M. D. Rosen, R. J. Wallace, F. A. Weber

December 1, 2003

Physical Review Letters 
This document was prepared as an account of work sponsored by an agency of the United States Government. Neither the United States Government nor the University of California nor any of their employees, makes any warranty, express or implied, or assumes any legal liability or responsibility for the accuracy, completeness, or usefulness of any information, apparatus, product, or process disclosed, or represents that its use would not infringe privately owned rights. Reference herein to any specific commercial product, process, or service by trade name, trademark, manufacturer, or otherwise, does not necessarily constitute or imply its endorsement, recommendation, or favoring by the United States Government or the University of California. The views and opinions of authors expressed herein do not necessarily state or reflect those of the United States Government or the University of California, and shall not be used for advertising or product endorsement purposes.

This work was performed under the auspices of the U.S. Department of Energy by the University of California, Lawrence Livermore National Laboratory under Contract No. W-7405-Eng-48. 


\section{UCRL-JRNL-201210 Measurement of the absolute hohlraum wall albedo under ignition foot drive conditions}

O. S. Jones, S. H. Glenzer, L. J. Suter, R. E. Turner, K. M. Campbell, E. L. Dewald, B. A. Hammel, J. H. Hammer, R. L. Kauffman, O. L. Landen, M. D. Rosen, R. J. Wallace, F. A. Weber

Lawrence Livermore National Laboratory, Livermore, California 94551

Abstract

We present the first measurements of the absolute albedos of hohlraums made from gold or from high-Z mixtures. The measurements are performed over the range of radiation temperatures (70-100 eV) expected during the foot of an indirect-drive temporallyshaped ignition laser pulse, where accurate knowledge of the wall albedo (i.e. soft x-ray wall re-emission) is most critical for determining capsule radiation symmetry. We find that the gold albedo agrees well with calculations using the super transition array opacity model, potentially providing additional margin for ICF ignition. 
Indirect drive inertial confinement fusion (ICF) uses high intensity lasers or particle beams to heat a high-Z cavity (hohlraum) that produces soft $\mathrm{x}$-rays characterized by a radiation temperature, $\mathrm{T}_{\mathrm{RAD}}[1]$. The $\mathrm{x}$-rays heat and ablate the surface of a DT-filled fusion capsule, compressing and heating the DT fuel until it ignites. Current indirect drive target designs predict that ignition and gain can be achieved by heating hohlraums with a temporally-shaped 1.5-2 MJ, 0.35-micron laser pulse [2-5]. The baseline ignition laser pulse creates a time-dependent $\mathrm{T}_{\mathrm{RAD}}$, or "drive", that consists of a 10-ns long "foot" at 80-100 eV, followed by a rapid increase in $\mathrm{T}_{\mathrm{RAD}}$ to a peak of $300 \mathrm{eV}$ at $15 \mathrm{~ns}$. The hohlraum conditions during the long foot of the pulse, including the uniformity of the radiation flux, are important. The level of the foot's $T_{\text {RAD }}$ sets the strength of the first shock, while any spatial illumination non-uniformities during the foot lead to asymmetric drive.

The albedo is the ratio of the re-emitted x-ray flux to the incident x-ray flux. Accurate knowledge of this quantity is important to the ignition effort because it affects our estimates of the asymmetry during the foot. Radiation uniformity depends on a variety of parameters including the location of the laser spots on the hohlraum wall and the x-ray brightness of those spots relative to the soft x-ray re-emission from the unirradiated wall. Higher albedo leads to a larger ratio of wall re-emission to spot brightness, which reduces capsule asymmetries due to laser power imbalance and beam mispointing.

In this letter, we report on the first experiments that measure the absolute albedo of hohlraums made from several different high-Z materials at radiation temperatures close to the NIF foot radiation temperature. We use a novel experimental technique in which the high- $Z$ hohlraum from which we infer the albedo is heated by soft x-rays and not by a laser, thus eliminating x-ray conversion efficiency and laser backscatter as parameters in the data analysis [6]. Moreover, the same instrument measures the flux incident upon the high- $Z$ hohlraum wall and the flux re-emitted from the wall. This technique results in a measurement error in the albedo of only $+/-0.06$. We show that the albedo of gold inferred from the experiment matches well with predictions using the radiation hydrodynamics code, LASNEX [7], with opacities from the super transition array (STA) model $[8,9]$. The measured albedo is larger than predicted from calculations that use the average-atom XSN model [10] for the opacity. This is important because the original NIF laser power balance specifications $[11,12]$ were based on modeling of gold hohlraums that used the average-atom XSN approximation for the opacities, resulting in an albedo at the end of the $10 \mathrm{~ns}$, 
$80 \mathrm{eV}$ foot pulse that is $\sim 20 \%$ lower than predicted using STA opacities. Figure 1 shows the variation in the random component of the flux asymmetry onto an ignition capsule during the foot as a function of the random laser power imbalance and the wall albedo. The curves are obtained from a radiation viewfactor analysis. Note that the capsule flux asymmetry is plotted as a function of the rms variation in the power of the 48 NIF quads, which are each made up of four beams. The curve for the albedo of 0.65 , which is consistent with XSN estimates of the average albedo for a gold hohlraum during the foot of an ignition pulse, is taken directly from Ref. [12]. The allowable contribution from laser imbalance to the flux asymmetry during the foot is about 0.006 , which corresponds to a foot-averaged quad-to-quad imbalance of about $4 \%$, and is consistent with the NIF specification. The other curve is for a wall albedo of 0.80 , which is more representative of the average wall albedo estimated using STA opacities. We see that at an albedo of 0.80 , the radiation on the capsule should be $\sim 30 \%$ more uniform than previously estimated, providing additional margin for ignition.

The experiments to measure low $\mathrm{T}_{\mathrm{RAD}}$ albedo were performed at the Omega laser facility. The experimental setup is shown in Figure 2. The basic configuration (Fig. 2b) consisted of two attached hohlraums. The primary hohlraum ( $1600 \mu \mathrm{m}$ diameter, $1125 \mu \mathrm{m}$ length) produced the soft x-ray drive that heated the secondary hohlraum, whose wall re-emission was measured in order to infer the wall albedo. The primary hohlraum was heated on one side by 15 laser beams that delivered $6.5 \mathrm{~kJ}$ in a $1.5-\mathrm{ns}$ temporally-flat pulse. It reached a peak radiation temperature of $\sim 160 \mathrm{eV}$ and that radiation heated the larger secondary hohlraum. We tested secondary hohlraums (with $3440 \mu \mathrm{m}$ diameter, and $3750 \mu \mathrm{m}$ length) made of gold, uranium (alloyed with 14\% atomic fraction niobium), and a mixture (or "cocktail") [13-15] made up of 52\% uranium, $8 \%$ niobium, $20 \%$ gold, and $20 \%$ dysprosium. These secondary hohlraums reached a peak radiation temperature of $\sim 100 \mathrm{eV}$. We also tested a larger gold secondary hohlraum (with $4400 \mu \mathrm{m}$ diameter, and $4800 \mu \mathrm{m}$ length), which had a lower peak radiation temperature $(\sim 70 \mathrm{eV})$ than the smaller secondary hohlraums. All secondary hohlraums had a $1000 \mu \mathrm{m}$ diameter diagnostic hole on the end.

The radiation power out of the secondary hohlraum diagnostic hole $\left(\mathrm{P}_{\mathrm{d}}\right)$ was measured by the Dante filtered x-ray diode (XRD) array [16] and also by a photoconductive detector (PCD) [17]. To obtain a direct measurement of the power leaving the primary hohlraum and heating the secondary hohlraum (the 
radiation source power, $\mathrm{P}_{\mathrm{s}}$ ), we also shot the primary hohlraum without the secondary attached (Fig. 2a), so that the power from the primary $\left(\mathrm{P}_{\mathrm{s}}\right)$ could be measured with the same two instruments as $\mathrm{P}_{\mathrm{d}}$. The principal measurement from this experiment is the ratio of the radiation power per solid angle onto the primary hohlraum XRD to the radiation power per solid angle onto the secondary hohlraum XRD. We shall now show that this ratio depends on the albedo of the secondary hohlraum wall.

A radiation power balance model $[18,19]$ for the secondary hohlraum can be formulated as

$$
P_{s}=P_{h}+P_{d}+P_{w}
$$

where $\mathrm{P}_{\mathrm{s}}$ is the source radiation power coming from the primary hohlraum, and $\mathrm{P}_{h}, \mathrm{P}_{\mathrm{d}}$, and $\mathrm{P}_{\mathrm{w}}$ are the radiation sinks leaving the secondary hohlraum through the hole between the two hohlraums, through the diagnostic hole, and into the wall, respectively (see Fig. 2). We define the average radiation temperature in the secondary hohlraum, $\mathrm{T}_{\mathrm{RAD}}$, by equating the total frequency-integrated radiation flux inside the hohlraum to a blackbody flux $\sigma \mathrm{T}_{\mathrm{RAD}}{ }^{4}$, where $\sigma$ is the Stefan-Boltzmann constant. The radiation power leaving the secondary through holes $\left(\mathrm{P}_{\mathrm{h}}\right.$ and $\left.\mathrm{P}_{\mathrm{d}}\right)$ is simply the radiation flux inside the secondary $\left(\sigma \mathrm{T}_{\mathrm{RAD}}{ }^{4}\right)$ times the hole areas $\left(A_{h}\right.$ and $\left.A_{d}\right)$, respectively. The remaining term, $P_{w}$, depends on the fraction of the impinging radiation that is absorbed by the wall (i.e. what is not absorbed is re-emitted and therefore not lost). We define the albedo, $\alpha$, as the ratio of the emitted radiation flux $\left(\mathrm{F}_{\text {out }}\right)$ to the incident radiation flux $\left(F_{i n}\right)$. Since the flux re-emitted by the wall is proportional to $\alpha$, the radiation power absorbed by the wall is (1- $\alpha)$ times the wall area $\left(\mathrm{A}_{\mathrm{w}}\right)$. When $\mathrm{P}_{\mathrm{h}}, \mathrm{P}_{\mathrm{d}}$, and $\mathrm{P}_{\mathrm{w}}$ are expressed in terms of $\mathrm{T}_{\mathrm{RAD}}$ and $\alpha$ and substituted into equation (1), we find that

$$
P_{s}=\sigma T_{R A D}^{4}\left[A_{h}+A_{d}+A_{w}(1-\alpha)\right]
$$

The radiation temperature of the secondary hohlraum $\left(\mathrm{T}_{\mathrm{RAD}}\right)$ in equation (2) is inferred from the measured power per steradian from the secondary hohlraum $\left(\mathrm{P}_{\mathrm{XRD} 2}\right)$ by noting that the XRD measures the re-emitted flux from the secondary wall, whereas $\sigma \mathrm{T}_{\mathrm{RAD}}{ }^{4}$ in equation (2) is the flux incident on the wall, related to the re-emitted flux via the albedo. We can express the re-emitted flux as

$$
\alpha \sigma T_{R A D}{ }^{4}=\alpha F_{\text {in,avg }}=\frac{1}{C_{2}} \alpha F_{\text {in,view }}=\frac{1}{C_{2}} \frac{P_{X R D 2} \pi}{A_{d} \sin \beta}
$$


where $\beta$ is the XRD view angle relative to the wall normal (see Fig. 2), $\mathrm{F}_{\text {in,avg }}$ is the average incident flux over the entire secondary hohlraum wall $\left(\mathrm{A}_{\mathrm{w}}\right), \mathrm{F}_{\text {in,view }}$ is the incident flux over the portion of the secondary hohlraum wall viewed by the XRD, and $\mathrm{C}_{2}$ is the factor that corrects for the nonuniformity of the incident flux. We are able to use the angle-averaged albedo in this equation because the viewing angle $\beta$ of $52.6^{\circ}$ is close to $48^{\circ}$, which is the angle at which the angle-dependent and angle-averaged albedos are equal. We calculated the spatial variation of the incident flux using a radiation viewfactor code. We find that $C_{2}$ is well approximated as a quadratic function of the secondary hohlraum albedo for both sizes of secondary hohlraum. That is, we have $C_{2}(\alpha)=1.927-0.834 \alpha-0.065 \alpha^{2}$ for the $3440 \mu \mathrm{m}(100 \mathrm{eV})$ secondary and $C_{2}(\alpha)=2.044-0.946 \alpha-0.080 \alpha^{2}$ for the $4400 \mu \mathrm{m}(70 \mathrm{eV})$ secondary.

The total source power $\left(\mathrm{P}_{\mathrm{s}}\right)$ is related to the power per steradian measured by the XRD in the primary-hohlraum shot $\left(\mathrm{P}_{\mathrm{XRD} 1}\right)$ by

$P_{s}=C_{1} P_{X R D 1} \frac{\pi}{\sin \beta}$

where $C_{1}$ is a factor that accounts for the angle-dependence of the emission due to the XRD viewing a combination of laser spots, unirradiated wall, and laser entrance hole (see Fig. 2). Here we neglect the contribution to the source power from the radiation coming back from the secondary hohlraum because the correction is small $(<5 \%)$ in comparison to the measurement uncertainty. From a Lasnex calculation of the gold primary hohlraum we find that $\mathrm{C}_{1}$ is close to 1 , but decreases with time due to hole closure and changing emission volume as the wall blow-off plasma in the primary hohlraum expands. We approximate $\mathrm{C}_{1}(\mathrm{t})$ as a piecewise linear function of time where $\mathrm{C}_{1}=1$ from 0 to $0.5 \mathrm{~ns}$, varies linearly between 1 and 0.88 from $0.5 \mathrm{~ns}$ to $1.5 \mathrm{~ns}$, and then varies linearly between 0.88 to 0.85 from $1.5 \mathrm{~ns}$ to $2.0 \mathrm{~ns}$.

Substituting equations (3) and (4) into (2), we get the following equation relating the albedo to the experimentally measured quantities:

$$
\left.\frac{P_{X R D 2}}{P_{X R D 1}}=\frac{\alpha C_{1}(t) C_{2}(\alpha)}{1+\frac{A_{h}}{A_{d}}+\frac{A_{w}}{A_{d}}(1-\alpha)}\right]
$$

We determined from Lasnex calculations of the double hohlraum system that equation (5) is self-consistent to within about $3 \%$, indicating that equation (5) is appropriate for extracting the hohlraum albedo from the 
experimental measurements. Figure 3 shows the secondary-to-primary XRD power ratio as a function of time for a $3440 \mu \mathrm{m}$ diameter gold secondary hohlraum. The measured data are the squares, with $20 \%$ error bars, which is the estimated uncertainty in unfolding the absolute radiation per solid angle from the 10 channel XRD array. The curves are created from the ratio of synthetic XRD powers per solid angle extracted from two Lasnex calculations: a primary-only calculation and a double hohlraum calculation. For one calculation STA opacities are used for the secondary hohlraum, leading to the upper curve which agrees well with the data. The lower curve is from an almost identical set of calculations, except that XSN opacities are used in the secondary. The curves begin to diverge from one another at about $0.7 \mathrm{~ns}$, and by 2 ns, the XSN curve has dropped outside the measured error bars.

A clearer distinction between the STA and XSN opacity models for gold can be seen by comparing the secondary-to-primary ratios of a single XRD channel, rather than comparing the total power ratios obtained from a spectral deconvolution of all 10 XRD channels. Rosen [20] has previously shown the inaccuracy of the XSN opacity model for gold at 70-100 eV by examining burn-through measurements. He noted that the inaccuracy is attributable to the lack of $n=4$ to 4 transitions near photon energies of 200-300 $\mathrm{eV}$ in the XSN average atom approximation. These transitions are included in the more detailed STA opacity model. The second XRD channel covers this important spectral range near the Planckian peak, from $~ 200-280 \mathrm{eV}$. Figure 4 compares the measured and calculated XRD channel 2 signal ratios (secondary to primary) for the $100 \mathrm{eV}$ gold secondary hohlraum. The ratio extracted from the STA calculation agrees with the data and is a factor of 2 above the XSN value, providing clear experimental verification that STA is needed to accurately model gold at this radiation temperature.

Figure 5 summarizes the data from all the different types of secondary hohlraums that were used in these experiments. The data are plotted in terms of an equivalent albedo at peak $\mathrm{T}_{\mathrm{RAD}}(\mathrm{t}=1.5 \mathrm{~ns})$ by substituting the secondary-to-primary XRD power ratio into equation (5). The measured albedos are known to within $+/-0.06$, which follows from equation (5), assuming a $20 \%$ uncertainty in the measured secondary-to-primary XRD ratio. Note that the absolute albedos plotted in Fig. 5 are lower than the albedos at the end of the $10 \mathrm{~ns}$ ignition foot pulse (see Fig. 1) because the Omega laser's energy limited these experiments to $1.5 \mathrm{~ns}$. The measured albedos are compared to the predicted albedos from the simulations that used STA opacities in the secondary hohlraum. The predicted albedos are also obtained 
from equation (5), but using the simulated secondary-to-primary XRD ratio instead of the measured ratio. For the gold and cocktail hohlraums, we see good agreement between the data and the STA prediction. We note here that the cocktail hohlraum does not have a higher albedo than gold at $100 \mathrm{eV}$ because the mixture of materials was chosen to maximize the albedo for radiation temperatures of 250-300 eV that occur during the peak of the ignition drive pulse. The only material that did not follow the expected trends was uranium, which has a measured albedo that is above the STA prediction. Direct measurements of the uranium opacity in this temperature regime will be required to determine whether STA is correctly predicting the opacity of uranium.

In summary, we have performed the first absolute measurements of the albedo of high-Z hohlraums in the temperature range of $70-100 \mathrm{eV}$, which is the range of temperatures that characterize the foot of an indirect drive ignition pulse. We find that the albedo of gold predicted from LASNEX calculations that use the STA opacity model agree well with the measured values, and that calculations using STA opacities provide better overall agreement with the data than calculations that use the average atom XSN model for the opacity. Since the gold albedo predicted for the foot of an ignition pulse using STA opacities is about $20 \%$ higher than previous XSN-based calculations, this results in approximately a $30 \%$ reduction in the random flux nonuniformity due to random laser power imbalance during the foot compared to previous estimates, providing additional margin for ignition. 


\section{References}

1. J. Lindl, Inertial Confinement Fusion, Springer-Verlag, New York, 1998

2. S. W. Haan, et al., Phys. Plasmas 2, 2480 (1995)

3. T. R. Dittrich, et al., Phys. Plasmas 6, 2164 (1999)

4. D. C. Wilson, et al., Phys. Plasmas 5, 1953 (1998)

5. $\quad$ W. J. Krauser, et al., Phys. Plasmas 3, 2084 (1996)

6. S. H. Glenzer, et al., Phys. Rev. Lett. 80, 2845 (1998)

7. G. B. Zimmerman and W. L. Kruer, Comments Plasma Phys. Controlled Fusion 2, 51 (1975).

8. $\quad$ A. Bar-Shalom, et al., Phys. Rev. A 40, 3183 (1989)

9. M. Klapisch, et al., Phys. Plasmas 8, 1817 (2001)

10. W. A. Lokke and W. H. Grasberger, LLNL Report UCRŁ 52276 (1977).

11. O. S. Jones, et al., 'The NIF Power Balance," Supplement to Proc. of SPIE Vol. 3492, 49 (1998).

12. O. S. Jones, et al., ICF Quarterly Report, 8, 140 (UCRL-LR-105821-98-4) (1998).

13. H. Nishimura, et al., Appl. Phys. Lett. 62, 1344 (1993)

14. T. J. Orzechowski, et al., Phys. Rev. Lett. 77, 3545 (1996)

15. D. Columbant, M. Klapisch, and A. Bar-Shalom, Phys. Rev. E 57, 3411 (1998)

16. H. N. Kornblum and R. L. Kauffman, Rev. Sci. Instrum. 57, 2179 (1986)

17. R. E. Turner, et al., Rev. Sci. Instrum. 70, 656 (1999)

18. L. Suter, et al., Phys. Plasmas 3, 2057 (1996)

19. R. L. Kauffmann, et al., Phys. Rev. Lett. 73, 2320 (1994)

20. M. D. Rosen, Phys. Plasmas 3, 1803 (1996) 
Figures

Fig. 1. Flux asymmetry onto ignition capsule during foot of pulse as function of random laser power imbalance and wall albedo.

Fig. 2. Schematic of the (a) primary and (b) double hohlraum experiment together with $\mathrm{x}$-ray power diagnostics and calculated x-ray emission.

Fig. 3. Measured (squares) and calculated (curves) time-dependent ratios of the secondary hohlraum power per solid angle to the primary hohlraum power per solid angle for a $3440 \mu \mathrm{m}$ diameter gold secondary hohlraum.

Fig. 4. Measured and predicted ratios of the secondary hohlraum XRD channel 2 (200$280 \mathrm{eV}$ ) signal to the primary XRD channel 2 signal.

Fig. 5. Comparison of the measured (squares) and predicted hohlraum albedos at peak $\mathrm{T}_{\mathrm{RAD}}$ for different secondary hohlraums. 


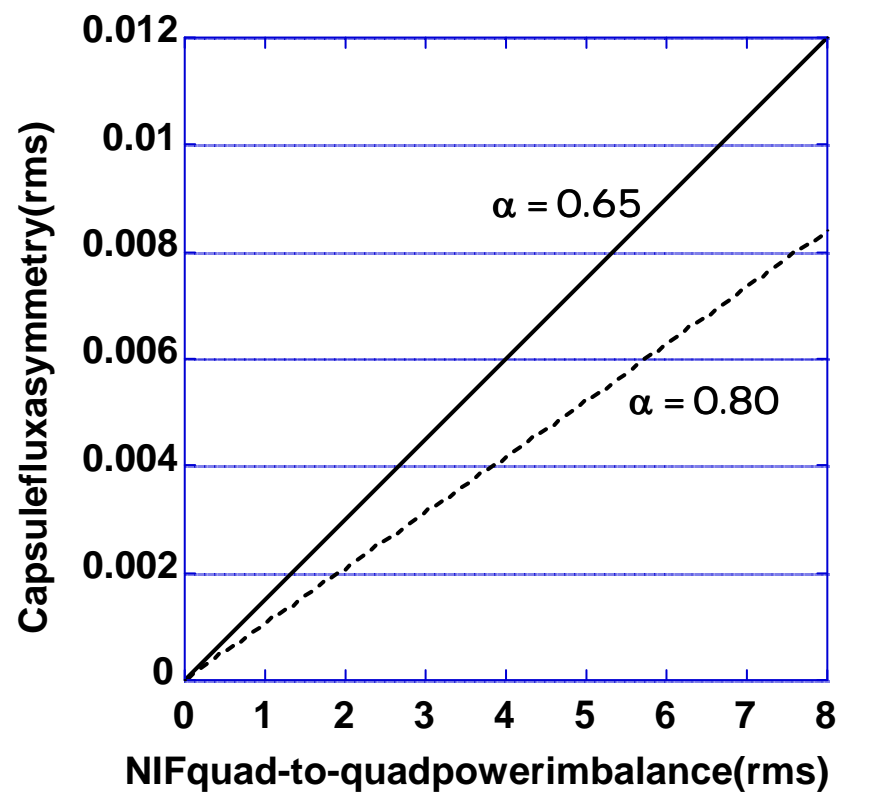

Fig. 1 


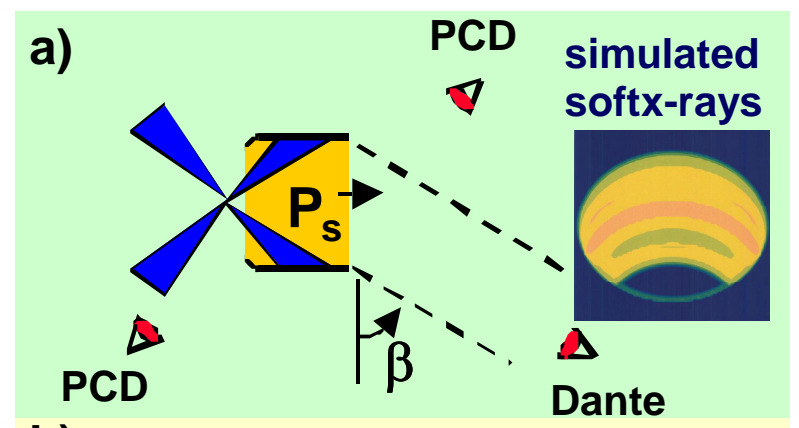

b)

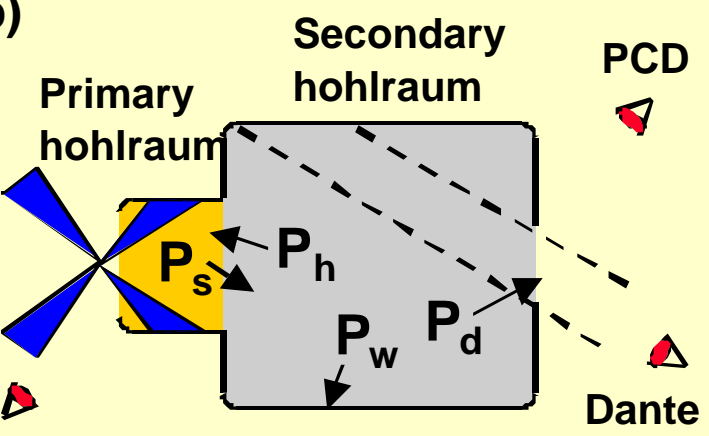

PCD

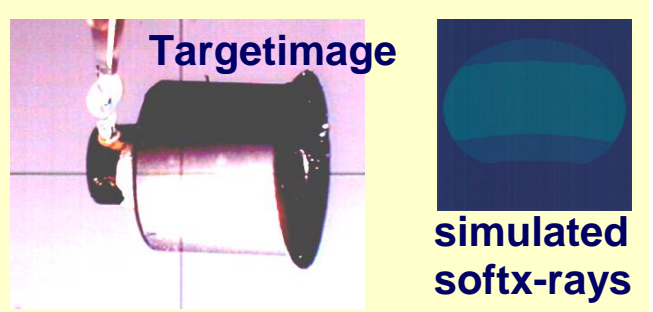

Fig. 2 


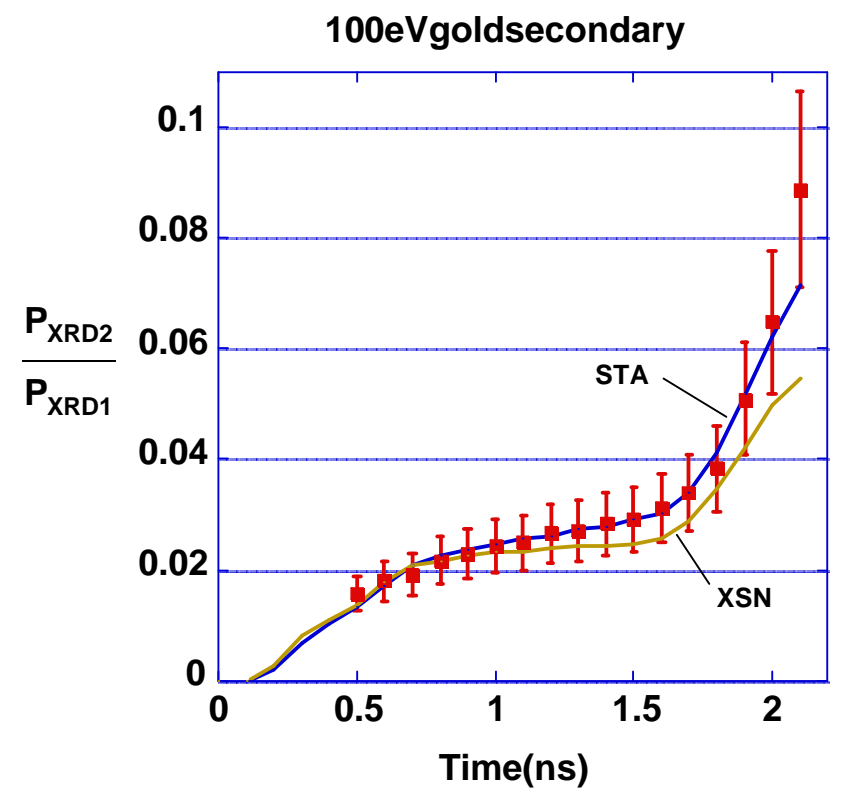

Fig. 3 


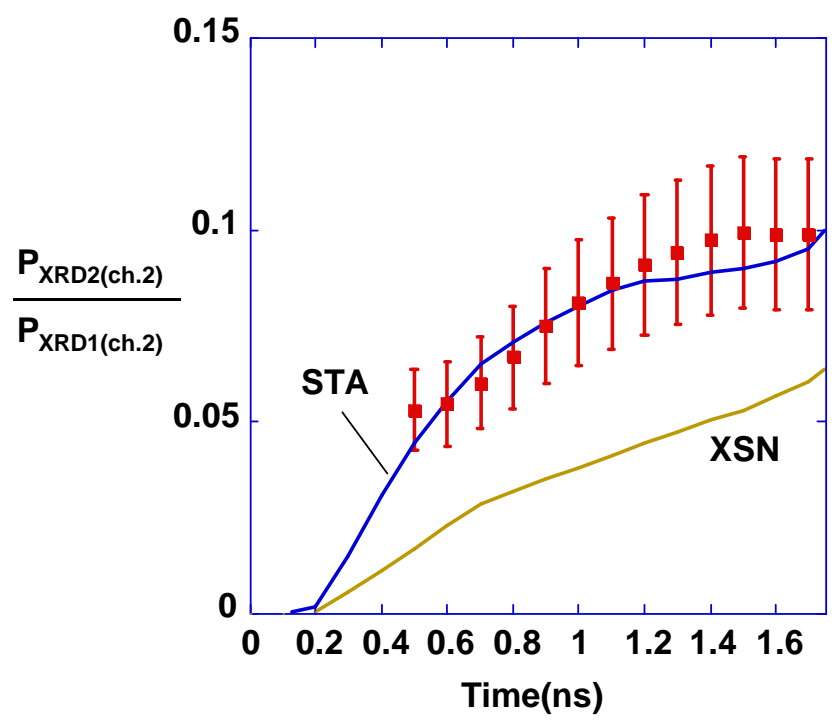

Fig. 4 


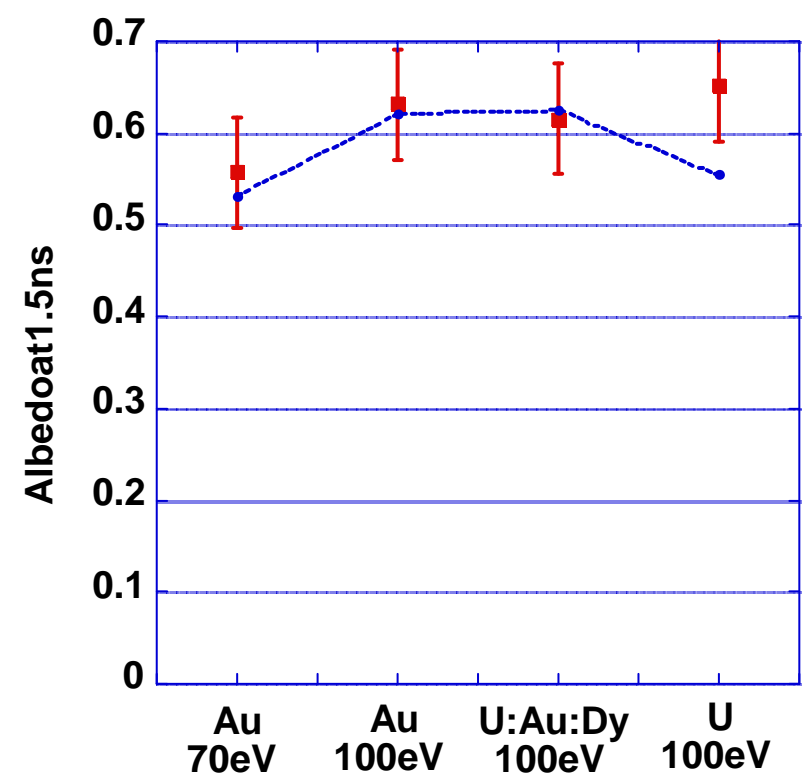

Fig. 5 
